\title{
Weak generic types and coverings of groups I
}

\author{
by \\ Ludomir Newelski and Marcin Petrykowski (Wrocław)
}

\begin{abstract}
We introduce the notion of a weak generic type in a group. We improve our earlier results on countable coverings of groups and types.
\end{abstract}

Introduction. Assume $G$ is an $\aleph_{0}$-saturated group, or even a group type-definable in an $\aleph_{0}$-saturated structure. Strictly speaking, by a group we sometimes mean an expansion of a pure group structure. Assume $G$ is covered by countably many 0 -type-definable sets $X_{n}, n<\omega$. In [NP] we proved that in this case some finitely many of the sets $X_{n}$ generate the group $G$ in at most $k=3$ steps. More precisely, we proved that

(C1) for some finite set $A \subseteq G$ and some $n<\omega$ we have $G=A \cdot X_{n} \cdot X_{n}^{-1}$. So in a sense for an arbitrary $\aleph_{0}$-saturated group $G, k=2.5$ steps suffice to generate $G$ by some finitely many of the sets $X_{n}, n<\omega$. In general, this result cannot be improved (that is, $k=2$ steps may not suffice). However, in $[\mathrm{NP}]$ we also proved that

(C2) if $G$ is abelian, then $G=X_{<n} \cdot X_{<n}^{-1}$ for some $n<\omega$, where $X_{<n}=$ $\bigcup_{i<n} X_{i}$.

Thus in this case $k=2$ steps are enough.

In this paper we give a new proof of $(\mathrm{C} 1)$, using the notion of a weak generic type in a group. This new notion generalizes that of a generic type in a stable group, fundamental in stable model theory. Besides its applicability in clarifying $(\mathrm{C} 1)$ we predict it may play an important role in model theory, particularly in unstable structures (like the o-minimal ones).

Regarding (C2), here we extend it in two ways. First, we generalize (C2) to any amenable group $G$, giving a completely new proof. Secondly, extending

2000 Mathematics Subject Classification: Primary 03C45. group.

Key words and phrases: generic type, weak generic type, coverings of groups, amenable

Research supported by KBN grant 2 P03A 01824. 
the proof of $(\mathrm{C} 2)$ from $[\mathrm{NP}]$, we prove a variant of $(\mathrm{C} 2)$ for an arbitrary group $G$. This raises a fascinating possibility of existence of a combinatorial counterpart of the Banach mean in any group. Unfortunately, thus far it is not clear to us what such a counterpart should be.

In $[N P]$ we also gave model-theory-free versions of $(\mathrm{C} 1)$ and $(\mathrm{C} 2)$. Here we do the same, giving model-theory-free versions of the results announced above. They are in fact equivalent to their model-theoretic counterparts, but less intuitive (at least to a model-theorist). Still, they may be appealing to a non-model-theorist.

Assume $p$ is a complete type over $\emptyset$ in a complete first order theory $T$. Let $M$ be an $\aleph_{0}$-saturated model of $T$. Assume $p(M) \times p(M)$ is covered by countably many 0 -type-definable sets $X_{n}, n<\omega$. We can regard $p(M)$ as the set of vertices of a complete directed graph $\Gamma$. Then the sets $X_{n}, n<\omega$, may be regarded as a colouring of the set of edges of $\Gamma$ into countably many colours (so that one edge may have more than one colour). In $[\mathrm{NP}]$ we proved that in this case

$\left(\mathrm{C} 1^{\prime}\right) \quad$ any $a, b \in p(M)$ may be connected by a path $a, c_{1}, c_{2}, b$ of length 3 so that the edges $\left\langle a, c_{1}\right\rangle,\left\langle c_{1}, c_{2}\right\rangle,\left\langle b, c_{2}\right\rangle$ lie in $X_{<n}$ for some $n<\omega$.

The proof in $[\mathrm{NP}]$ was a modification of the proof of $(\mathrm{C} 1)$ there.

Here we introduce the notion of a c-free and weakly c-free extension of a type, generalizing the notion of a non-forking extension in a stable theory. Using this notion we give a new proof of $\left(\mathrm{C}^{\prime}\right)$. The notion of a weakly c-free extension corresponds to that of a weak generic type in a group in the same manner as the proof of $\left(\mathrm{C}^{\prime}\right)$ in $[\mathrm{NP}]$ corresponds to the proof of $(\mathrm{C} 1)$ there. We predict it may have more applications in unstable structures.

Possible counterparts of the extensions of (C2) announced above (like the case of amenable groups) in the case of coverings of types are more problematic, and we do not pursue them here. We conclude the paper with a set of problems.

Throughout we use standard model-theoretic notation. The results on amenable groups are due to the second author, the rest of the paper is due to the first author.

We would like to thank Gabriel Sabbagh for interesting conversations on this paper.

1. Weak generic types. In this section we assume $G$ is an $\aleph_{0}$-saturated group, or even a type-definable group in an $\aleph_{0}$-saturated model of a complete first order theory $T$. When $G$ (or $T$ ) is stable, the notion of a generic type of elements of $G$ was introduced by Poizat [Po]. It became a fundamental notion in geometric model theory. In the stable case we can define generic types as follows [Wa, Pi]. 
Definition 1.1. We say that a set $X \subseteq G$ is (left) generic if some finitely many left $G$-translates of $X$ cover $G$. We say that a formula $\varphi(x)$ is (left) generic if the set $\varphi(G)$ of elements of $G$ realizing $\varphi$ is (left) generic. Finally, we say that a type $p(x)$ of elements of $G$ is (left) generic if every formula $\varphi(x)$ with $p(x) \vdash \varphi(x)$ is (left) generic. By default, "generic" always means "left generic".

In the stable case left generic $=$ right generic (defined by means of right translations) and each partial generic type extends to a complete generic type (over any set of parameters); moreover generic types may be characterized there by means of forking and translation-invariant ranks.

While Definition 1.1 is appealing by its simplicity, it does not work well in the unstable context. For instance, complete generic types in the sense of Definition 1.1 may not exist in general. So in simple theories generic types are defined by extending the definition from the stable case, which refers to forking and ranks. However, in this paper we do apply Definition 1.1 in the general setting, and to overcome its drawbacks we define a weaker notion, still capturing the sense of being a "not-so-small" subset of $G$. Suppose $X=A \cup B$ is a generic subset of $G$. If $B$ is not generic, then one could argue that $A$ is "not-so-small". This justifies the following definition.

Definition 1.2. We say that a set $A \subseteq G$ is weak generic if $A \cup B$ is generic for some non-generic $B \subseteq G$. We say that a formula $\varphi(x)$ is weak generic if the set $\varphi(G)$ is weak generic. A type $p(x)$ of elements of $G$ is weak generic if every formula $\varphi(x)$ with $p(x) \vdash \varphi(x)$ is weak generic.

We are interested mainly in the definable (weak) generic sets. For these purposes, the next lemma provides an alternative definition. In the case where $G$ is type-definable in an $\aleph_{0}$-saturated structure $M$, in the next lemma and also throughout the paper by a "definable" subset of $G$ we mean a "relatively definable" subset of $G$.

Lemma 1.3. Assume $U$ is a definable subset of $G$. Then the following conditions are equivalent.

(1) $U$ is weak generic.

(2) For some finitely many elements $a_{0}, \ldots, a_{n-1} \in G$, the set $\bigcap_{i<n} a_{i} U^{\mathrm{c}}$ is not generic.

(3) For some definable non-generic set $V \subseteq G$, the set $U \cup V$ is generic.

Proof. (1) $\Rightarrow(2)$. Assume $U$ is weak generic. Hence for some non-generic set $V \subseteq G$, the set $U \cup V$ is generic, meaning that for some finitely many elements $a_{0}, \ldots, a_{n-1} \in G$ we have

$$
\bigcup_{i<n} a_{i}(U \cup V)=\bigcup_{i<n} a_{i} U \cup \bigcup_{i<n} a_{i} V=G .
$$


This means that

$$
\bigcap_{i<n} a_{i} U^{\mathrm{c}} \subseteq \bigcup_{i<n} a_{i} V
$$

Since $V$ is not generic, neither is $\bigcup_{i<n} a_{i} V$, and we are done.

$(2) \Rightarrow(3)$. Let $V=\bigcap_{i<n} a_{i} U^{\mathrm{c}}$. We see that $V$ is definable, not generic, and putting $a_{n}=e$ (the neutral element of $\left.G\right)$, we find that $G=\bigcup_{i \leq n} a_{i}(U \cup V)$, hence $U \cup V$ is generic.

$(3) \Rightarrow(1)$ is trivial.

In the next lemma we give some basic properties of weak generic sets and types.

LEMMA 1.4.

(1) If $U_{1}, U_{2} \subseteq G$ are not weak generic, then $U_{1} \cup U_{2}$ is not weak generic.

(2) Every (partial) weak generic type over $A \subseteq G$ can be extended to a complete weak generic type over A.

Proof. (1) Let $V \subseteq G$ be non-generic. Since $U_{2}$ is not weak generic, $U_{2} \cup V$ is not generic. Since $U_{1}$ is not weak generic, $U_{1} \cup U_{2} \cup V$ is not generic. Hence $U_{1} \cup U_{2}$ is not weak generic.

(2) follows from (1).

We see that weak generic types exist. By Lemma 1.4, the set

$$
\operatorname{WGen}(A)=\{p \in S(A): p \text { is weak generic }\}
$$

is closed and non-empty in $S(A)$. The next lemma explains the relationship between weak generic types and generic types, provided the latter exist.

LEMMA 1.5 .

(1) If some weak generic type $p(x) \in S(G)$ is generic, then all weak generic types $q(x) \in S(A)$ are generic.

(2) If there is just one weak generic type in $S(A)$, then it is generic.

Proof. (1) Suppose some weak generic type $q(x) \in S(A)$ is not generic. Extending $q$ to a weak generic type over $G$ we can assume $A=G$. Then some definable generic set $X \subseteq G$ can be divided into two non-generic definable sets $A, B$. Since $X$ is generic, some left $G$-translate $X^{\prime}$ of $X$ belongs to $p(x)$. Then the corresponding translates $A^{\prime}, B^{\prime}$ of $A, B$ are also non-generic, and one of them belongs to $p(x)$, hence $p(x)$ is not generic.

(2) A similar proof.

By Lemma 1.5, in the stable case weak generic $=$ generic (for types and definable sets). As an example notice that if $G=(G, \cdot,<, \ldots)$ is o-minimal, then there are exactly two complete weak generic types, corresponding to $+\infty$ and $-\infty$, and they are not generic. Hence in (2) the assumption that there is only one weak generic type cannot be weakened. Description of 
weak generic types in other groups definable in o-minimal structures is more complicated; it will appear in a forthcoming paper by the second author.

The next proposition shows that weak genericity is related to generating $G$. We identify sets of types with subsets of the model consisting of the elements realizing the types from these sets. That is, if $P \subseteq S(\emptyset)$, then sometimes we identify $P$ with the set $P(G)=\bigcup\{p(G): p \in P\}$. Also, if $P$ is open (closed, Borel etc.), then we call the set $P(G)$ open (closed, Borel, etc.). In particular, a closed subset of $G$ is just a 0-type-definable one, and a clopen subset of $G$ is just a 0 -definable one. For a formula $\varphi(x),[\varphi]$ denotes the class of types containing $\varphi$. We adopt a similar notation for types.

Proposition 1.6. Assume $P \subseteq \mathrm{WGen}(\emptyset)$ is non-empty and relatively open. Then for some finite $A \subseteq G$ we have $G=A \cdot P(G) \cdot P(G)^{-1}$.

Proof. Choose a non-empty 0-definable subset $U$ of $G$ with $P \supseteq$ WGen $(\emptyset)$ $\cap[U] \neq \emptyset$. We can assume that $P=\mathrm{WGen}(\emptyset) \cap[U]$. Hence $U$ is weak generic. Choose a definable non-generic set $V \subseteq G$ such that $U \cup V$ is generic. Choose a finite set $A \subseteq G$ with $G=A \cdot(U \cup V)$. We prove that

$$
\text { the set }(U \cup V) \backslash P(G) \text { is not generic. }
$$

Suppose not. Then there is a finite set $B \subseteq G$ with $B \cdot((U \cup V) \backslash P(G))$ $=G$. By compactness, for some 0-definable set $W$ with $P(G) \subseteq W \subseteq U$ we have $B \cdot((U \cup V) \backslash W)=G$, i.e. the set $(U \cup V) \backslash W$ is generic. Since $(U \cup V) \backslash W \subseteq V \cup(U \backslash W)$, it follows that the set $U \backslash W$ is weak generic. But $[U \backslash W] \cap$ WGen $(\emptyset)=\emptyset$, contradicting Lemma 1.4(2).

To finish the proof, we show that $G=A \cdot P(G) \cdot P(G)^{-1}$, that is, for every $g \in G$ there is some $h \in P(G)$ with $g \cdot h \in A \cdot P(G)$.

If not, then there is some $g \in G$ such that for every $h \in P(G)$ we have $g \cdot h \notin A \cdot P(G)$. Still $g \cdot h \in A \cdot(U \cup V)$, hence $g \cdot h \in A \cdot((U \cup V) \backslash P(G))$ and $\left.P(G) \subseteq g^{-1} \cdot A \cdot((U \cup V)) \backslash P(G)\right)$. We see that

$$
U \cup V=((U \cup V) \backslash P(G)) \cup P(G) \subseteq\left(\{e\} \cup g^{-1} \cdot A\right) \cdot((U \cup V) \backslash P(G)),
$$

and $G=A \cdot\left(\{e\} \cup g^{-1} \cdot A\right) \cdot((U \cup V) \backslash P(G))$, contradicting $(*)$.

Using Proposition 1.6 we may give a new proof of [NP, Theorem 2.1], which we restate as Corollary 1.7 below.

Corollary 1.7. Assume an $\aleph_{0}$-saturated group $G$ is covered by some 0 -type-definable sets $X_{n}, n<\omega$. Then for some finite $A \subseteq G$ and some $n<\omega$ we have $G=A \cdot X_{n} \cdot X_{n}^{-1}$.

Proof. At the level of types, $S(\emptyset)$ is covered by countably many closed sets $\left[X_{n}\right], n<\omega$. Hence by the Baire category theorem, for some $n$, the set $\left[X_{n}\right] \cap \mathrm{WGen}(\emptyset)$ has non-empty relative interior in WGen $(\emptyset)$. We apply Proposition 1.6. 
If the theory of $G$ is simple, generic types are usually defined via forking. Namely, we say that a formula $\varphi(x, a)$ is generic in $G$ if for every $b \in G$, the formula $\varphi(b \cdot x, a)$ does not fork over $\emptyset$ (see [Wa]). Here we will call a formula with this property $f$-generic, to distinguish it from the notion from Definition 1.1. Similarly, we call a type $f$-generic if every formula it implies is f-generic. The next lemma shows that in simple theories "weak generic" and "f-generic" are related.

Lemma 1.8. If $\operatorname{Th}(G)$ is simple and $\varphi(x, a)$ is weak generic in $G$, then $\varphi(x, a)$ is f-generic.

Proof. We may assume $a=\emptyset$. Let $V=\varphi(G)$ and choose a non-generic definable set $U \subseteq G$ with $U \cup V$ generic. Choose a finite set $A \subseteq G$ with $A \cdot(U \cup V)=G$. Suppose for contradiction that $\varphi(x)$ is not f-generic. Then each translate of $V$ and also any union of finitely many such translates is not f-generic (see [Wa]). Let $\psi(x)$ be a formula defining the set $A \cdot V$. Since $\psi$ is not f-generic, some left translate of $\psi$ forks over $\emptyset$. In simple theories, forking $=$ dividing, hence we find an infinite indiscernible sequence $b_{i}, i<\omega$, with $\bigcap_{i} b_{i} \cdot A \cdot V=\emptyset$. So, for some $n<\omega$ we have $\bigcap_{i<n} b_{i} \cdot A \cdot V=\emptyset$.

To reach a contradiction, we will prove that $U$ is generic. We know that $A \cdot(U \cup V)=G$. Hence also $b_{i} \cdot A \cdot U \cup b_{i} \cdot A \cdot V=G$ for every $i<n$. Thus

$$
G \backslash \bigcup_{i<n} b_{i} \cdot A \cdot U \subseteq \bigcap_{i<n} b_{i} \cdot A \cdot V=\emptyset,
$$

meaning that $\bigcup_{i<n} b_{i} \cdot A \cdot U=G$, a contradiction.

Usually in the simple unstable case, generic types (in the sense of Definition 1.1) do not exist in a group $G$; however, weak generic types do, and by Lemma 1.8 they form a closed subset of the set of f-generic types. As usual with simple theories, we know of no example where in a group $G$ with simple theory an f-generic formula is not weak generic.

2. The case where two steps suffice. Again assume $G$ is an $\aleph_{0^{-}}$ saturated group, covered by countably many 0-type-definable sets $X_{n}, n<\omega$. In $[\mathrm{NP}]$ and in Section 1 we showed that in general $G$ as a group is generated by some finitely many of the sets $X_{n}$ in $k=2.5$ steps. Sometimes $k=2$ steps suffice, that is, for some $n, G=X_{<n} \cdot X_{<n}^{-1}$, where $X_{<n}=\bigcup_{i<n} X_{i}$. In [NP] we proved that it is so when $G$ is abelian or stable. Here we improve this result by replacing "abelian" with "amenable". Also, we give a uniform proof for both the amenable and stable cases.

Recall [W] that a group $G$ is amenable if there exists a finitely additive measure $\mu$ on $\mathcal{P}(G)$ such that $\mu(G)=1$ and $\mu$ is left-invariant (i.e. $\mu(g \cdot A)=$ $\mu(A)$ for every $g \in G$ and $A \subseteq G)$. We call any such $\mu$ a Banach mean on $G$. Amenable groups form a large class, including solvable groups, and more 
generally all groups without a paradoxical decomposition. However, no nonabelian free group is amenable. Any stable group is also "amenable" in the following weak sense.

Proposition 2.1. Assume $G$ is stable. Then there is a finitely additive left-invariant measure $\mu$ with $\mu(G)=1$, defined on the algebra $\operatorname{Def}(G)$ of all definable subsets of $G$.

Proof. Let $\mathcal{G}$ be the set of generic types of $G$ (over acl ${ }^{\mathrm{eq}}(\emptyset)$ ). As pointed out in [Ne1], $\mathcal{G}$ is a compact topological group, with the topology induced from $S\left(\operatorname{acl}^{\mathrm{eq}}(\emptyset)\right)$. The group operation is the independent multiplication of types: $r_{1} * r_{2}=\operatorname{stp}\left(a_{1} \cdot a_{2}\right)$, where $a_{1}$ and $a_{2}$ are independent realizations of $r_{1}, r_{2}$ respectively. The generic type of the connected component $G^{0}$ of $G$ is the identity element of $\mathcal{G}$. Let $\nu$ be the Haar measure on $\mathcal{G}$. Then $\nu$ induces a measure $\mu$ on $\operatorname{Def}(G)$ as follows. Let $\varphi(x, a)$ be a formula. We associate with $\varphi$ the set

$$
V_{\varphi}=\{r \in \mathcal{G}: \varphi(x, a) \text { belongs to the non-forking extension of } r\} .
$$

It is a relatively clopen subset of $\mathcal{G}$. We define $\mu(\varphi(G, a))$ as $\nu\left(V_{\varphi}\right)$. This works.

The proof of Proposition 2.1 shows that in fact $\mu$ may be extended to the $\sigma$-algebra of sets generated by $\operatorname{Def}(G)$, and $\mu$ is $\sigma$-additive. We will not need this, however. We will use the following lemma on finitely additive measures.

Lemma 2.2. Assume $X$ is a set and $\mu$ is a finitely additive finite measure on an algebra $\mathcal{A}$ of subsets of $X$. Assume for some $\varepsilon>0$ we have a family $A_{n}$, $n<\omega$, of sets from $\mathcal{A}$ with $\mu\left(A_{n}\right)>\varepsilon$ for all $n$. Then for some increasing sequence $\left(n_{k}\right)_{k<\omega}$ of natural numbers, and each $i<\omega$,

$$
\mu\left(A_{n_{0}} \cap \cdots \cap A_{n_{i}}\right)>0 .
$$

Proof. First we prove that under the assumptions of the lemma,

$(*) \quad$ there are $n<\omega$ and $\varepsilon^{\prime}>0$ such that the set $\left\{m: \mu\left(A_{n} \cap A_{m}\right)>\varepsilon^{\prime}\right\}$ is infinite.

If not, for every $n<\omega$ and $\varepsilon^{\prime}>0$, the set $\left\{m: \mu\left(A_{n} \cap A_{m}\right)>\varepsilon^{\prime}\right\}$ is finite. Let $k$ be a positive integer such that $\mu\left(A_{n}\right)>2 / k$ for every $n<\omega$. Put $n_{0}=0$ and for each $i=1, \ldots, k-1$ choose recursively $n_{i}<\omega$ so that for all $j<i, \mu\left(A_{n_{i}} \cap A_{n_{j}}\right)<1 /(k i)$. We have

$$
1 \geq \mu\left(\bigcup_{i=0}^{k-1} A_{n_{i}}\right)=\sum_{i=0}^{k-1} \mu\left(A_{n_{i}} \backslash \bigcup_{j<i} A_{n_{j}}\right)>\sum_{i=0}^{k-1}\left(\frac{2}{k}-\frac{1}{k}\right)=1,
$$

a contradiction. 
Having proved $(*)$, we define recursively numbers $n_{k}, k<\omega$, so that for each $k<\omega$ we have

(**) for some $\varepsilon^{\prime}>0$, the set $Z=\left\{m: \mu\left(A_{n_{0}} \cap \cdots \cap A_{n_{k}} \cap A_{m}\right)>\varepsilon^{\prime}\right\}$ is infinite.

For $k=0$ we define $n_{0}$ by $(*)$. For the recursion step, suppose we have defined $n_{0}, \ldots, n_{k}$ so that $(* *)$ holds. We will define $n_{k+1}$. Let $X^{\prime}=A_{n_{0}} \cap \cdots \cap A_{n_{k}}$ and for $m \in Z$ set $A_{m}^{\prime}=X^{\prime} \cap A_{m}$. We see that $X^{\prime}$ and $A_{m}^{\prime}, m \in Z$, satisfy the assumptions of the lemma for the restricted $\mu$, so that in this situation $(*)$ holds. Let $n_{k}$ be the $n$ furnished by $(*)$. This finishes the construction and the proof.

THEOREM 2.3. If $G$ is stable or amenable, and $G$ is covered by countably many 0-type-definable sets $X_{n}, n<\omega$, then $G=X_{<n} \cdot X_{<n}^{-1}$ for some $n<\omega$.

Proof. Stability and amenability of $G$ together imply that there exists a finitely additive left-invariant measure $\mu$ on $\operatorname{Def}(G)$ with $\mu(G)=1$. For the sake of contradiction assume that for any $n<\omega$ we can find $a_{n} \in G \backslash X_{<n}$. $X_{<n}^{-1}$. By compactness there is a formula $\varphi_{n}(x)$ such that $X_{<n} \subseteq \varphi_{n}(G)$ and $a_{n} \notin \varphi_{n}(G) \cdot \varphi_{n}(G)^{-1}$. We have

$$
G=\neg \varphi_{n}(G) \cup a_{n} \cdot \neg \varphi_{n}(G) .
$$

Since $\mu\left(\neg \varphi_{n}(G)\right)=\mu\left(a_{n} \cdot \neg \varphi_{n}(G)\right)$, it follows that $\mu\left(\neg \varphi_{n}(G)\right) \geq 1 / 2$ for each $n<\omega$. By Lemma 2.2 we are able to choose an increasing sequence $\left(n_{k}\right)_{k<\omega}$ with

$$
\mu\left(\neg \varphi_{n_{0}}(G) \cap \cdots \cap \neg \varphi_{n_{k}}(G)\right)>0
$$

for every $k<\omega$. On the other hand, $\left\{\varphi_{n_{k}}(G): k<\omega\right\}$ is a family of definable sets covering $G$, so we can choose a finite subcovering $\left\{\varphi_{n_{k}}(G): k<K\right\}$ (for some $K<\omega)$. Then $\mu\left(\bigcap_{k<K} \neg \varphi_{n_{k}}(G)\right)=\mu(\emptyset)=0$, a contradiction.

In particular, the conclusion of Theorem 2.3 holds when $G$ is abelian (since each abelian group is amenable). This special case was already proved in [NP]. However, the proof of Theorem 2.3 is completely different. The proof in [NP] is in a way more direct and it does not refer to such an ambiguous and ineffective object as a Banach mean on a group. One could wonder if it is possible to extend the proof from $[\mathrm{NP}]$ to deal not only with abelian groups, but also with some other amenable groups, like for instance the nilpotent or solvable ones. We have found however another striking way to generalize the proof from [NP]. For $X \subseteq G$ and $a, x \in G$, let $x^{a}$ denote $a^{-1} x a$ and $X^{a}=\left\{x^{a}: x \in X\right\}$. We have the following theorem.

Theorem 2.4. Assume $G$ is an $\aleph_{0}$-saturated group covered by countably many 0-type-definable sets $X_{n}, n<\omega$. Then for some finite set $A \subseteq G$ and 
some $n<\omega$ we have

$$
G=\bigcup_{a \in A}\left(X_{<n} \cdot X_{<n}^{-1}\right)^{a},
$$

that is, $G$ is covered by the set of a-conjugates of $X_{<n} \cdot X_{<n}^{-1}, a \in A$.

When $G$ is amenable, Theorem 2.4 follows from Theorem 2.3 (with $A$ $=\{e\})$. Before the proof we recall a construction from $[\mathrm{Ne} 2, \mathrm{NP}]$.

Assume $X$ is a compact topological space covered by a family $\mathcal{A}$ of subsets of $X$. We define an increasing sequence $Z_{\alpha}, \alpha \in\{-1\} \cup$ Ord, of open subsets of $X$. We put $Z_{-1}=\emptyset, Z_{\alpha+1}=\bigcup_{A \in \mathcal{A}} \operatorname{int}\left(Z_{\alpha} \cup A\right)$ and for limit $\alpha, Z_{\alpha}=$ $\bigcup_{\beta<\alpha} Z_{\beta}$. The sequence $\left(Z_{\alpha}\right)$ is called the open analysis of $X$ with respect to $\mathcal{A}$. By the Baire category theorem if $\mathcal{A}$ is a countable family of closed sets covering $X$, then $Z_{\alpha}=X$ for some $\alpha$.

Proof. The proof of Theorem 2.4 will be a modification of the proof of [NP, Theorem 3.1], where $G$ is abelian. Let $Y=S(\emptyset)$ and $Y_{n}=S(\emptyset) \cap$ $\left[X_{n}(x)\right]$. Let $Z_{\alpha}, \alpha \in\{-1\} \cup \operatorname{Ord}$, be the open analysis of $Y$ with respect to $Y_{n}, n<\omega$. So $Z_{\alpha}=Y$ for some $\alpha$. For a closed set $C \subseteq Y$ let $\gamma(C)$ be the minimal ordinal (or -1 ) such that $C \subseteq Z_{\gamma(C)}$. So $\gamma(\emptyset)=-1$ and by compactness, for each closed $C, \gamma(C)$ equals $-1,0$, or is a successor ordinal.

Suppose the theorem fails. We define recursively 0-definable sets $A_{n} \subseteq G$, elements $a_{n} \in G$ and closed sets $C_{n} \subseteq Y, n<\omega$, so that the following hold.

(a) $C_{n}=\left\{\operatorname{tp}(a): a \in A_{n}\right\}$.

(b) For every $U \subseteq n, a_{U} a_{n} a_{U}^{-1}$ is not of the form $h_{1} h_{2}^{-1}$ for any $h_{1}, h_{2} \in$ $G \backslash A_{n}$. Here $a_{U}=a_{i_{0}} \cdots a_{i_{k-1}}$, where $i_{0}<\cdots<i_{k-1}$ is the increasing enumeration of $U$. For $U=\emptyset$ we stipulate $a_{U}=e$, the neutral element of $G$.

(c) For all $k<\omega$ and all $i_{0}<\cdots<i_{k}<\omega$, if $Y \cap C_{i_{0}} \cap \cdots \cap C_{i_{k-1}} \neq \emptyset$, then

$$
\gamma\left(Y \cap C_{i_{0}} \cap \cdots \cap C_{i_{k}}\right)<\gamma\left(Y \cap C_{i_{0}} \cap \cdots \cap C_{i_{k-1}}\right) .
$$

Assume we have defined $A_{i}, C_{i}, a_{i}$ for all $i<n$. We choose $N<\omega$ so that for every $k<\omega$ and all $i_{0}<\cdots<i_{k}<\omega$ with $Y \cap C_{i_{0}} \cap \cdots \cap C_{i_{k-1}} \neq \emptyset$,

$$
\left(Y \cap C_{i_{0}} \cap \cdots \cap C_{i_{k-1}}\right) \backslash Z_{\gamma-1} \subseteq Y_{<N},
$$

where $\gamma=\gamma\left(Y \cap C_{i_{0}} \cap \cdots \cap C_{i_{k-1}}\right)$.

Let $A=\left\{a_{U}: U \subseteq n\right\}$. Since $G \neq \bigcup_{a \in A}\left(X_{<N} X_{<N}^{-1}\right)^{a}$, by compactness there is a formula $\varphi(x)$ over $\emptyset$ such that $X_{<N} \subseteq \varphi(G)$ and

$$
G \neq \bigcup_{a \in A}\left(\varphi(G) \varphi(G)^{-1}\right)^{a} .
$$

We put $A_{n}=G \backslash \varphi(G)$, choose $a_{n} \in G \backslash \bigcup_{a \in A}\left(\varphi(G) \varphi(G)^{-1}\right)^{a}$ and define $C_{n}$ accordingly. Then (a) and (b) hold. The choice of $N$ ensures (c). 
Since there is no infinite decreasing sequence of ordinals, the family of closed sets $C_{n}, n<\omega$, has no infinite subfamily with the finite intersection property. It follows that

(d) Every infinite set $I \subseteq \omega$ has a finite subset $I^{\prime}$ with $\bigcap_{n \in I^{\prime}} A_{n}=\emptyset$. Now we sketch the idea of the proof. If the sequence $\left(A_{n}, a_{n}\right)_{n<\omega}$ is orderindiscernible with respect to all relations

$$
a_{i_{0}} \cdots a_{i_{k-1}} \in A_{j} \quad\left(i_{0}<\cdots<i_{k-1}<\omega, j<\omega\right),
$$

then we easily reach a contradiction. Namely, for $j \notin\left\{i_{0}, \ldots, i_{k-1}\right\}$, by (d) we have

$$
h_{2}=a_{i_{0}} a_{i_{1}} \cdots a_{i_{k-1}} \notin A_{j} .
$$

Say, $i_{0}<\cdots<i_{l-1}<j<i_{l}<\cdots<i_{k-1}$. If also $h_{1}=a_{i_{0}} \cdots a_{i_{l-1}} a_{j} a_{i_{l}} \cdots$ $a_{i_{k-1}} \notin A_{j}$, then

$$
h_{1} h_{2}^{-1}=a_{i_{0}} \cdots a_{i_{l-1}} a_{j} a_{i_{l-1}}^{-1} \cdots a_{i_{0}}^{-1}
$$

would contradict (b). So $a_{i_{0}} \cdots a_{i_{l-1}} a_{j} a_{i_{l}} \cdots a_{i_{k-1}} \in A_{j}$. By indiscernibility, $a_{0} \cdots a_{n} \in A_{0} \cap \cdots \cap A_{n}$ for all $n<\omega$, contradicting (d) again.

This reasoning would lead to a contradiction even if we had an infinite subsequence of $\left(A_{n}, a_{n}\right)_{n<\omega}$ order-indiscernible in the above sense. However, since here we need indiscernibility with respect to infinitely many relations, we cannot hope for such a subsequence to exist. Instead, in [NP] we introduce a notion of $(\alpha, k)$-indiscernibility (where $\alpha<\omega_{1}, k<\omega$ ), which approximates full order-indiscernibility.

Let $\alpha$ be the order type of the set

$$
\Omega=\left\{\gamma\left(Y \cap C_{i_{0}} \cap \cdots \cap C_{i_{k-1}}\right): k<\omega \text { and } i_{0}<\cdots<i_{k-1}<\omega\right\} .
$$

Since $\Omega$ is countable, $\alpha<\omega_{1}$. Now we work with an $(\alpha, 0)$-indiscernible subsequence of $\left(A_{n}, a_{n}\right)_{n<\omega}$, reaching a contradiction precisely as in [NP].

The proofs of Theorem 2.4 and its predecessor, [NP, Theorem 3.1], remain mysterious to us. Theorem 2.3 suggests that in the special case where $G$ is abelian, Theorem 2.4 just tells us something about the amenability of $G$. But Theorem 2.4 is about an arbitrary group, possibly non-amenable. This suggests a possibility of finding a combinatorial counterpart of the Banach mean in an arbitrary group. This may require improving Theorem 2.4 in a way that we will try to suggest now.

Corollary 1.7 says that for some finite $A \subseteq G$ and some $n^{*}<\omega$ we have $G=A \cdot X_{n^{*}} \cdot X_{n^{*}}^{-1}$. Our feeling is that this means that $X_{n^{*}}$ is large in some sense. Now suppose we refine our covering, presenting each $X_{n}$ as a union of countably many 0 -type-definable sets $X_{n, k}, k<\omega$. If $X_{n^{*}}$ is large, then one would expect that one of the sets $X_{n^{*}, k}$ should also be large, that is, for some finite $A^{\prime} \subseteq G$ and some $k<\omega$ we should have $G=A^{\prime} \cdot X_{n^{*}, k} \cdot X_{n^{*}, k}^{-1}$. This may not be true. 
For example, one can construct recursively an infinite subset $B$ of $\mathbb{Z}$ such that every non-zero integer can be uniquely presented as $a-b$, where $a, b \in B$. Moreover, we can ensure that $B$ can be split into two disjoint sets $B_{0}, B_{1}$ such that for both sets $B_{0}-B_{0}$ and $B_{1}-B_{1}$ there are arbitrarily long intervals disjoint from them. Consider the structure $(\mathbb{Z},+)$ expanded by the predicates $B, B_{0}, B_{1}$. Let $G$ be an $\aleph_{0}$-saturated elementary extension of $\left(\mathbb{Z},+, B, B_{0}, B_{1}\right)$. Let $X_{0}=B(G)$, and $X_{1}=B(G)^{\mathrm{c}}$. Then $X_{0}-X_{0}=G$. Let $X_{0,0}=B_{0}(G)$ and $X_{0,1}=B_{1}(G)$. Then $X_{0}=X_{0,0} \cup X_{0,1}$, but for every finite $A \subseteq G$ we have $G \neq A+X_{0, i}-X_{0, i}, i=0,1$.

However, the current proof of Corollary 1.7 suggests the right notion of largeness here. Namely, we can consider a 0-type-definable set $X \subseteq G$ to be large if WGen $\cap[X]$ has non-empty interior in WGen. Then if $X_{n^{*}}$ is large, then so is $X_{n^{*}, k}$ for some $k$.

Now assume $G$ is amenable. By Theorem 2.3, $G=X_{<n^{*}} \cdot X_{<n^{*}}^{-1}$ for some $n^{*}$. This suggests that $X_{<n^{*}}$ is large in some sense. This should mean that refining our covering $X_{n}, n<\omega$, to a covering $X_{n, k}, n, k<\omega$ (as above), it should be the case that for some $k^{*}<\omega$, the set $X_{<n^{*},<k^{*}}=$ $\bigcup_{i<n^{*}} \bigcup_{j<k^{*}} X_{i, j}$ is large, meaning that $G=X_{<n^{*},<k^{*}} \cdot X_{<n^{*},<k^{*}}^{-1}$.

This may fail. For example, assume $G$ has a definable subgroup $H$ of index 2. Let $X_{1}=H \backslash\{e\}$ and $X_{0}=G \backslash X_{1}$. Then $G=X_{0} \cdot X_{0}^{-1}$. However, if we split $X_{0}$ into infinitely many non-empty sets $X_{0, k}, k<\omega$, then it is not true that $G=X_{0,<k} \cdot X_{0,<k}^{-1}$ for some $k$.

We can correct this as follows. Assume $\mu$ is a Banach mean on $G$. For a subset $X \subseteq G$ define the "exterior" measure $\mu^{*}(X)$ by

$$
\mu^{*}(X)=\inf \{\mu(U): X \subseteq U \subseteq G \text { and } U \text { is 0-definable }\} .
$$

We say that $X$ is large if $\mu^{*}(X)>1 / 2$. Then one sees that if $X$ is large and 0 -type-definable, then $G=X \cdot X^{-1}$. Actually, the set $X_{0}$ from the above example is not large, since $\mu^{*}\left(X_{0}\right)=1 / 2$. A counterpart of Lemma 2.2 holds for $\mu^{*}$ in place of $\mu$. Using this we see that if $G$ is covered by 0-type-definable sets $X_{n}, n<\omega$, then for some $n^{*}$ we have $\mu^{*}\left(X_{<n^{*}}\right)>1 / 2$, i.e. $X_{<n^{*}}$ is large (and $G=X_{<n^{*}} \cdot X_{<n^{*}}^{-1}$ ). This notion is hereditary: if we consider a refinement $X_{n, k}, n, k<\omega$, of our covering and $X_{<n^{*}}$ is large, then for some $k^{*}$ we have $\mu^{*}\left(X_{<n^{*},<k^{*}}\right)>1 / 2$ (whence $G=X_{<n^{*},<k^{*}} \cdot X_{<n^{*},<k^{*}}^{-1}$ ).

We do not see how to make Theorem 2.4 hereditary in the same manner. We believe that finding a way to do so could reveal a notion of "largeness" which would be a remote combinatorial counterpart of the Banach mean in an arbitrary group.

Another argument for a possibility of generalizing the Banach mean is as follows. In the special case of abelian group our original complicated proof of Theorem 2.4 from [NP] was replaced here by an easier proof, using the Banach mean. Now, we have an equally complicated proof of Theorem 2.4. 
It is possible that a simplification of this proof (if it exists) would involve a general counterpart of the Banach mean.

Theorem 2.4 has an interesting corollary. Thus far we have considered arbitrary 0-type-definable sets $X_{n} \subseteq G$, possibly in a language larger than that of pure groups. For pure groups we can say more.

Corollary 2.5. Assume $X_{n} \subseteq G, n<\omega$, are 0-type-definable in the pure group language. Then $G=X_{<n} \cdot X_{<n}^{-1}$ for some $n<\omega$.

Proof. By Theorem 2.4, for some $n$, some finitely many conjugates of $X_{<n} \cdot X_{<n}^{-1}$ cover $G$. But since we are in the pure group structure, for any $a \in G$ we have $X_{<n}^{a}=X_{<n}$, so we are done.

3. Model-theory-free versions. In this section $G$ is an arbitrary infinite group, $X$ is a compact topological space and $f: G \rightarrow X$ is any function such that $f[G]$ is dense in $X$. In this setting we prove the counterparts of the results from Sections 1 and 2. First we define the notion of [weak] genericity in this new context.

Assume $U \subseteq X$. We call $U$ generic if $G=A \cdot f^{-1}[U]$ for some finite set $A \subseteq G$. We call $U$ weak generic if for some non-generic $V \subseteq X$, the set $U \cup V$ is generic. We call a point $p \in X[$ weak $]$ generic if every open set $U$ containing $p$ is [weak] generic. Let Gen and WGen be the sets of generic and weak generic points of $X$, respectively. The next lemma has a similar proof to Lemma 1.4.

LEMMA 3.1.

(1) If $U_{1}, U_{2} \subseteq X$ are not weak generic, then $U_{1} \cup U_{2}$ is not weak generic.

(2) Both WGen and Gen are closed subsets of $X$, Gen $\subseteq$ WGen and WGen is non-empty.

(3) Every open set $U$ containing WGen is generic.

Proof. For example, we will prove (3). Since no point in $X \backslash U$ is weak generic, by compactness we can find finitely many non-weak-generic open sets $V_{0}, \ldots, V_{k}$ (for some $k<\omega$ ) such that $U \cup V_{0} \cup \cdots \cup V_{k}=X$. Then by (1) also $V=V_{0} \cup \cdots \cup V_{k}$ is open and not weak generic, and still $U \cup V=X$. Since $X$ is generic, so is $U$.

For example, if $G$ is a compact topological group, $X=G$ and $f=$ id, then any point $p \in G$ is generic. Generic points not always exist, and this is why we deal with weak generic points instead. For example, assume $G$ is the additive group of the reals, $X=G \cup\{+\infty,-\infty\}$ is a 2-point compactification of $G$, and $f=$ id. Then $-\infty$ and $+\infty$ are the only weak generic points of $X$, and they are not generic. Our idea behind this definition is that if $p \in X$ is [weak] generic, then the group $G$ is "concentrated" around $p$ more than around a non-[weak]-generic point. Also, we think that investigating the 
set of weak generic points reveals how similar the triple $(G, X, f)$ is to the case of a compact topological group. The next proposition corresponds to Proposition 1.6.

Proposition 3.2. Assume $S$ is a non-empty, relatively open subset of WGen. Then there exists a finite set $A \subseteq G$ such that $G=A \cdot f^{-1}[U]$. $\left(f^{-1}[U]\right)^{-1}$ for every open set $U \supseteq S$.

Proof. Using normality of $X$ choose a relatively open non-empty subset $S^{\prime}$ of $S$ with $\operatorname{cl}\left(S^{\prime}\right) \subseteq S$. Then $\operatorname{cl}\left(S^{\prime}\right)$ and WGen $\backslash S$ are closed disjoint sets, hence there is an open set $V \subseteq X$ such that $\operatorname{cl}\left(S^{\prime}\right) \subseteq V$ and $\operatorname{cl}(V) \cap$ WGen $\subseteq S$.

Since $V$ is weak generic, there is a non-generic set $W \subseteq X$ such that $V \cup W$ is generic. Choose a finite set $A \subseteq G$ with $G=A \cdot f^{-1}[V \cup W]$. We will prove that $A$ satisfies our demands.

So let $U \supseteq S$ be open. By normality (working in the subspace $U \cup V$ ), there is an open set $V^{\prime} \subseteq V$ such that

$$
\operatorname{cl}\left(V^{\prime}\right) \cap U \cap \mathrm{WGen}=\emptyset \text { and } \quad U \cup V=U \cup V^{\prime} .
$$

Since

$$
\operatorname{cl}\left(V^{\prime}\right) \cap \text { WGen } \subseteq \operatorname{cl}(V) \cap \text { WGen } \subseteq S \subseteq U \cap \text { WGen, }
$$

we see that $\operatorname{cl}\left(V^{\prime}\right) \cap \mathrm{WGen}=\emptyset$. This means that $V^{\prime}$ is not weak generic, hence $V^{\prime} \cup W$ is not generic.

We will prove that $G=A \cdot f^{-1}[U] \cdot\left(f^{-1}[U]\right)^{-1}$. If not, then there is some $g \in G$ such that for every $h \in f^{-1}[U]$ we have $g \cdot h \notin A \cdot f^{-1}[U]$. Still $g \cdot h \in A \cdot f^{-1}[V \cup W]$, hence $g \cdot h \in A \cdot f^{-1}\left[V^{\prime} \cup W\right]$ and $h \in g^{-1} \cdot A \cdot f^{-1}\left[V^{\prime} \cup W\right]$. We see that $f^{-1}[U] \subseteq g^{-1} \cdot A \cdot f^{-1}\left[V^{\prime} \cup W\right]$, hence

$$
f^{-1}[V \cup W] \subseteq f^{-1}\left[V^{\prime} \cup W\right] \cup f^{-1}[U] \subseteq\left(\{e\} \cup g^{-1} \cdot A\right) \cdot f^{-1}\left[V^{\prime} \cup W\right]
$$

and $G=A \cdot f^{-1}[V \cup W]=A \cdot\left(\{e\} \cup g^{-1} \cdot A\right) \cdot f^{-1}\left[V^{\prime} \cup W\right]$. So $V^{\prime} \cup W$ is generic, a contradiction.

Corollary 3.3. Assume $f: G \rightarrow X$, where $G$ is a group and $X$ is a compact topological space covered by countably many closed sets $X_{n}, n<\omega$. Then there exist a finite set $A \subseteq G$ and $n<\omega$ such that for every open set $U \supseteq X_{n}$ we have $G=A \cdot f^{-1}[U] \cdot\left(f^{-1}[U]\right)^{-1}$.

Proof. By the Baire category theorem, for some $n$ the set $S=X_{n} \cap$ WGen has non-empty relative interior in WGen, hence the conclusion follows from Proposition 3.2.

Proposition 1.6 and Corollary 1.7 follow from Proposition 3.2 and Corollary 3.3. Namely assume for a moment that $G$ is $\aleph_{0}$-saturated. Then we can take $X=S(\emptyset)$ and $f: G \rightarrow X$ may be defined by $f(a)=\operatorname{tp}(a)$. Then the notion of a [weak] generic type translates to that of a [weak] generic point, and a covering of $G$ by 0 -type-definable sets corresponds to a covering of 
$X$ by closed sets, whence Proposition 3.2 and Corollary 3.3 apply. However, they are not really stronger than Proposition 1.6 and Corollary 1.7.

To be more specific, consider the following situation. Assume that besides $f: G \rightarrow X$ we have yet another function $f^{\prime}: G \rightarrow X^{\prime}$, where $X^{\prime}$ is also a compact topological space with $f^{\prime}[G]$ dense in $X^{\prime}$, and there is a continuous function $h^{\prime}: X^{\prime} \rightarrow X$ such that the following diagram commutes:

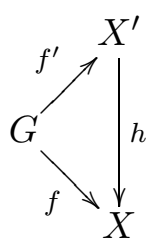

Let Gen' and WGen' denote the sets of generic and weak generic points of $X^{\prime}$, respectively (with respect to $f^{\prime}: G^{\prime} \rightarrow X^{\prime}$ ).

LEMMA 3.4.

(1) WGen $\subseteq h\left[\mathrm{WGen}^{\prime}\right]$.

(2) If $h$ restricted to $f^{\prime}[G]$ is $1-1$, then WGen $=h\left[\mathrm{WGen}^{\prime}\right]$.

(3) Gen $\supseteq h\left[\mathrm{Gen}^{\prime}\right]$.

Proof. (1) Assume $p \in \mathrm{WGen} \backslash h\left[\mathrm{WGen}^{\prime}\right]$. Choose an open $U \ni p$ with $\operatorname{cl}(U) \cap h\left[\mathrm{WGen}^{\prime}\right]=\emptyset$. Let $U^{\prime}=h^{-1}[U]$. Then $U^{\prime}$ is open and $\operatorname{cl}\left(U^{\prime}\right) \cap$ $\mathrm{WGen}^{\prime}=\emptyset$. It follows that $U$ is weak generic and $U^{\prime}$ is not weak generic. Choose a non-generic $W \subseteq X$ with $U \cup W$ generic. Let $W^{\prime}=h^{-1}[W]$. Then $U^{\prime} \cup W^{\prime}=h^{-1}[U \cup W]$ is generic. Since $U^{\prime}$ is not generic, we infer that $W^{\prime}$ is generic, hence also $W$ is generic, a contradiction.

(2), (3) Similar proofs.

The largest possible $X^{\prime}$ we can take in the above diagram (so that $h$ is $1-1$ on $f^{\prime}[G]$ ) is the space $\beta(f[G])$ (the space of ultrafilters on $f[G]$ ). Then $f^{\prime}: G \rightarrow X^{\prime}$ is the function induced by $f$, and there is a natural continuous function $h: X^{\prime} \rightarrow X$ such that the above diagram commutes. By Lemma $3.4(2), h\left[\mathrm{WGen}^{\prime}\right]=$ WGen. In this situation $X^{\prime}$ is 0 -dimensional. We expand $G$ by predicates $\left(f^{\prime}\right)^{-1}[U]$, where $U \subseteq X^{\prime}$ is clopen. Then WGen' (hence also WGen) is a continuous image of the set of weak generic types of the $\aleph_{0}$-saturated extension of this expansion of $G$. In this way Proposition 3.2 and Corollary 3.3 follow from Proposition 1.6 and Corollary 1.7.

The largest possible $X$ in $f: G \rightarrow X$ is the space $\beta(G)$ of ultrafilters on $G$ (and then $f$ maps $g \in G$ to the principal ultrafilter generated by $g$ ). Then no point in $X$ is generic. The other extreme is the case where $X$ is a singleton or $X=G$ and $f=$ id (in the case where $G$ is a compact topological group). Then any point is generic. 
Although the non-model-theoretic formulation of our results may be of independent interest, we think that the model-theoretic presentation is more elegant here. The following theorem is slightly stronger than its modeltheoretic version.

Theorem 3.5. Assume $f: G \rightarrow X$, where $G$ is an amenable group and $X$ is a compact space covered by countably many sets $X_{n}, n<\omega$ (not necessarily closed). Then there is an $n<\omega$ such that for every open set $U \supseteq \bigcup_{i<n} X_{i}$ we have $G=f^{-1}[U] \cdot\left(f^{-1}[U]\right)^{-1}$.

Proof. Let $\mu$ be a Banach mean on $G$. For the sake of contradiction assume that for every $n<\omega$ we can find an open set $U_{n} \supseteq X_{<n}$ such that $G \neq f^{-1}\left[U_{n}\right] \cdot\left(f^{-1}\left[U_{n}\right]\right)^{-1}$. Let $A_{n}=G \backslash f^{-1}\left[U_{n}\right]$ and choose $a_{n} \in G \backslash$ $f^{-1}\left[U_{n}\right] \cdot\left(f^{-1}\left[U_{n}\right]\right)^{-1}$.

Now if $g \in G$, then either $g \notin f^{-1}\left[U_{n}\right]$ or $a_{n}^{-1} \cdot g \notin f^{-1}\left[U_{n}\right]$, which means that $G=A_{n} \cup a_{n} \cdot A_{n}$. Since $\mu\left(A_{n}\right)=\mu\left(a_{n} \cdot A_{n}\right)$, we have $\mu\left(A_{n}\right) \geq 1 / 2$ for each $n<\omega$.

By Lemma 2.2 we are able to choose an increasing sequence $\left(n_{k}\right)$ of natural numbers with $\mu\left(A_{n_{0}} \cap \cdots \cap A_{n_{k}}\right)>0$ for every $k<\omega$. On the other hand, $\left\{U_{n_{k}}: k<\omega\right\}$ is a family of open sets covering the compact space $X$, so we can choose a finite subcovering $\left\{U_{n_{k}}: k<K\right\}$. Then $\left\{f^{-1}\left[U_{n_{k}}\right]: k<K\right\}$ is a finite family of sets covering $G$, so $A_{n_{0}} \cap \cdots \cap A_{n_{K}}=\emptyset$. This implies that $\mu\left(A_{n_{0}} \cap \cdots \cap A_{n_{K}}\right)=0$, a contradiction.

The next theorem corresponds to Theorem 2.4.

Theorem 3.6. Assume $f: G \rightarrow X$, where $G$ is a group and $X$ is a compact space covered by countably many closed sets $X_{n}, n<\omega$. Then there are a finite set $A \subseteq G$ and a natural number $n$ such that for every open set $U$ containing $X_{<n}$ we have

$$
G=\bigcup_{a \in A}\left(f^{-1}[U] \cdot\left(f^{-1}[U]\right)^{-1}\right)^{a} .
$$

Proof. The proof is similar to that of Theorem 2.4. Suppose the theorem fails. Let $Z_{\alpha}, \alpha \in\{-1\} \cup$ Ord, be the open analysis of $X$ with respect to $X_{n}, n<\omega$. For a closed set $C \subseteq X$ let $\gamma(C)$ be the minimal number such that $C \subseteq Z_{\gamma(C)}$. We define recursively closed sets $C_{n} \subseteq X$ and elements $a_{n} \in G, n<\omega$, so that the following hold.

(a) For every $V \subseteq n, a_{V} a_{n} a_{V}^{-1} \notin f^{-1}\left[X \backslash C_{n}\right] \cdot\left(f^{-1}\left[X \backslash C_{n}\right]\right)^{-1}$ (here $a_{V}$ is defined as in the proof of 2.4).

(b) For all $k<\omega$ and all $i_{0}<\cdots<i_{k}<\omega$ with $X \cap C_{i_{0}} \cap \cdots \cap C_{i_{k-1}} \neq \emptyset$ we have

$$
\gamma\left(X \cap C_{i_{0}} \cap \cdots \cap C_{i_{k}}\right)<\gamma\left(X \cap C_{i_{0}} \cap \cdots \cap C_{i_{k-1}}\right) .
$$


Having defined $C_{i}$ and $a_{i}$ for each $i<n$ we want to define $C_{n}$ and $a_{n}$. The crucial point of the recursive step is as follows. For a large natural number $N$ (chosen so as to satisfy (b)), there is an open set $U \supseteq X_{<N}$ such that

$$
G \neq \bigcup_{a \in A}\left(f^{-1}[U] \cdot\left(f^{-1}[U]\right)^{-1}\right)^{a},
$$

where $A=\left\{a_{V}: V \subseteq n\right\}$. Then we put $C_{n}=X \backslash U$ and choose

$$
a_{n} \in G \backslash \bigcup_{a \in A}\left(f^{-1}[U] \cdot f^{-1}[U]^{-1}\right)^{a} .
$$

The rest of the proof is as before.

4. An example. In this section we are again interested in an $\aleph_{0}$-saturated group $G$ covered by countably many 0-type-definable sets $X_{n}, n<\omega$. Suppose $G \backslash X_{<n} \cdot X_{<n}^{-1}$ is countable for some $n$. Usually in model theory a countable subset of an $\aleph_{0}$-saturated model is considered small. In particular, in our case, if $G \backslash X_{<n} \cdot X_{<n}^{-1}$ is countable, then $G \backslash X_{<n} \cdot X_{<n}^{-1} \subseteq \operatorname{acl}(\emptyset)$. So one could wonder if in this situation there is a possibly larger $n^{\prime}$ such that $G=X_{<n^{\prime}} \cdot X_{<n^{\prime}}^{-1}$. Here we give an example showing it may not be so.

Let $G$ be the free group with free generators $e_{n}, n \geq 0$. We regard elements of $G$ as irreducible words in letters $e_{n}^{ \pm 1}$. Let $\left\{v_{k}: k<\omega\right\}$ be an enumeration of $G$ (without repetitions) such that $\left|v_{k}\right| \leq k$. We define words $w_{k} \in G, k<\omega$, as follows.

- $w_{0}=e=\varepsilon$ (the empty word, the neutral element of $G$ ).

- $w_{2 k+1}=w_{2 k} \cdot e_{j}^{l}$, where $l=1+\sum_{i<2 k}\left|w_{i}\right|$ and $j \in\{0,1,2\}$ is chosen so that neither $w_{2 k}$ ends nor $v_{k}$ begins with $e_{j}^{ \pm 1}$.

- $w_{2 k+2}=w_{2 k+1} v_{k}$.

LEMMA 4.1.

(1) Each $w_{k}$ is an irreducible word.

(2) Each $w_{k}$ is an initial segment of $w_{k+1}$.

(3) Each $v_{k}$ equals $w_{l}^{-1} \cdot w_{t}$ for some $l, t$.

(4) Each $v_{k}$ equals $w_{l}^{-1} \cdot w_{t}$ for at most finitely many distinct $l, t<\omega$.

Proof. (1)-(3) are obvious. For (4) notice that if $l \neq t$, then in its irreducible form the word $w_{l}^{-1} w_{t}$ has length $\geq \max \{l, t\}$.

For each $w_{k}, k>0$, we define a derived sequence of words $w_{k, i}$ as follows. We present $w_{k}$ as $u v$, where $v$ is the longest suffix of $w_{k}$ in letters $e_{n}^{ \pm 1}, n>2$. Notice that $w_{k-1}$ is an initial segment of $u$. Write $v$ as $x_{l-1} x_{l-2} \cdots x_{0}$, where $l<\omega$ and $x_{i} \in\left\{e_{n}^{ \pm 1}: n>2\right\}$ ( $v$ may be empty, then $\left.l=0\right)$. We define $w_{k, i}$ as

$$
w_{k, i}=u x_{l-1} x_{l-2} \cdots x_{i} \quad \text { for } 0<i<l,
$$

and if $w_{k-1} \neq u$, then we additionally define $w_{k, l}$ as $u$. 
In this way none of the words $w_{k}, k<\omega$, is a derived word.

Lemma 4.2. If each of $u, v$ is derived or is one of the words $\left\{w_{k}: k \geq 0\right\}$, then one of $u, v$ is an initial segment of the other.

Now we define a countable partition of $G$ into sets $A_{n, m}$, where $n \geq-1$ and $m \geq 0$.

1. When $m<n$, we put $A_{m, n}=\emptyset$.

2. $A_{-1, m}=\left\{w_{m}\right\}$ for $m \geq 0$.

3. We define the sets $A_{n, m}, m \geq n \geq 0$, in two steps, assigning gradually all elements of $G$ distinct from $w_{m}, m \geq 0$, to one of them.

3 (a) Consider a word $w_{k}$, where $k>0$, presented as $u v$,

$$
v=x_{l-1} x_{l-2} \cdots x_{0}
$$

as in the definition of derived words. Assume $x_{l-1}$ is $e_{n}^{ \pm 1}$ (where $n>2)$. If $0 \leq i<l-1$ and $x_{i}$ is $e_{m}^{ \pm 1}(m>2)$, then we include $w_{k, i+1}$ into $A_{m^{\prime}, n^{\prime}}$, where $m^{\prime}=\min (m, n)$ and $n^{\prime}=\max (m, n)$. If $u \neq w_{k-1}$ and so also $w_{k, l}=u$ is defined, we put $w_{k, l}$ into $A_{n, n}$.

3(b) Assume a word $w \notin\left\{w_{k}: k \geq 0\right\}$ was not included into any of the sets $A_{n, m}$ in Step 3(a). Then $w$ begins with some $e_{n}^{ \pm 1}$ and ends with some $e_{m}^{ \pm 1}$. We include $w$ into $A_{m^{\prime}, n^{\prime}}$, where $m^{\prime}=\min (m, n)$ and $n^{\prime}=\max (m, n)$.

In this way we have defined a partition of $G$ into sets $A_{n, m}, n \geq-1, m \geq 0$. We call the words $w_{k}, k \geq 0$, special, and the words considered in Step 3(b) common.

Now we describe our example. We consider the countable compact space $X=\omega \cdot \omega+1$, with the order topology. We identify the set of isolated points of $X$ with the set of pairs $(n, m)$ of integers, where $n \geq-1$ and $m \geq 0$. More precisely, we identify $(-1, m)$ with $\alpha=m \in X$ and for $n \geq 0$ we identify $(n, m)$ with $\alpha=\omega(n+1)+(m+1)$. We can imagine the space $X$ as an infinite array:

$$
\begin{array}{ccccc}
(-1,0) & (-1,1) & (-1,2) & \ldots & \omega \cdot 1 \\
(0,0) & (0,1) & (0,1) & \ldots & \omega \cdot 2 \\
(1,0) & (1,1) & (1,2) & \ldots & \omega \cdot 3 \\
\vdots & \vdots & \vdots & & \vdots \\
& & & & \omega \cdot \omega
\end{array}
$$

where the points $\omega \cdot 1, \omega \cdot 2, \omega \cdot 3, \ldots$ are limit points in their rows, and $\omega \cdot \omega$ is the limit point of them. Then we consider a function $f: G \rightarrow X$ given by

$$
f(g)=(n, m) \Leftrightarrow g \in A_{n, m} .
$$

The example is an $\aleph_{0}$-saturated group $H$, an elementary extension of the 
group $G$ expanded by the predicates $f^{-1}[U]$, where $U$ ranges over the clopen subsets of $X$. To be more specific, we mention explicitly some of these predicates.

For an isolated $\alpha \in X$ let $P_{\alpha}(x)$ denote the predicate $f^{-1}[\{\alpha\}]$ in $G$. For $\alpha=\omega \cdot n, n>0$, let

$$
U_{\alpha}=\{\omega \cdot(n-1)+m: m>0\} \cup\{\omega \cdot n\}
$$

and let $P_{\alpha}(x)$ denote the predicate $f^{-1}\left[U_{\alpha}\right]$ in $G$.

We define 0-type-definable sets $X_{\alpha}, \alpha \in X$, in $H$. For an isolated $\alpha \in X$ we put $X_{\alpha}=P_{\alpha}(H)$ (so $X_{\alpha}$ is definable). When $\alpha=\omega \cdot n, n>0$, we put

$$
X_{\alpha}=P_{\alpha}(H) \backslash \bigcup\left\{P_{\beta}(H): \beta=\omega \cdot(n-1)+m \text { and } m>0\right\} .
$$

Finally, for $\alpha=\omega \cdot \omega$ we define $X_{\alpha}$ as $H \backslash \bigcup\left\{P_{\alpha}(H): \alpha=\omega \cdot n\right.$ and $\left.n>0\right\}$.

So $X_{\alpha}, \alpha \in X$, is a countable covering of $H$ by 0 -type-definable sets. We will show that $H \backslash X_{\omega}^{-1} \cdot X_{\omega}$ is countable.

Indeed, $X_{\omega}=\bigcap_{n} Y_{n}$, where

$$
Y_{n}=P_{\omega}(H) \backslash \bigcup_{0<m<n} A_{-1, m}
$$

Each $Y_{n}$ is a 0-definable subset of $H$. By Lemma 4.1(3),(4) we see that $\left(Y_{n} \cap G\right)^{-1} \cdot\left(Y_{n} \cap G\right)$ is a co-finite subset of $G$. Since $H$ is an elementary extension of the expansion of $G$, also $Y_{n}^{-1} \cdot Y_{n}$ is a co-finite subset of $H$. It follows that $X_{\omega}^{-1} \cdot X_{\omega}$ is a co-countable subset of $H$.

We will prove that

(*) $\quad H \neq X_{I}^{-1} \cdot X_{I}$ for every finite set $I \subseteq X$, where $X_{I}=\bigcup_{\alpha \in I} X_{\alpha}$.

This is equivalent to the fact that for every finite set $I \subseteq X$,

$$
G \neq\left(f^{-1}[U]\right)^{-1} \cdot f^{-1}[U] \text { for some open set } U \supseteq I .
$$

To prove $(* *)$, we can consider a finite set $I$ of the form

$$
I_{K}=\{(n, m): n, m<K\} \cup\{\omega \cdot n: 0<n \leq K\} \cup\{\omega \cdot \omega\},
$$

where $K<\omega$, since any finite subset of $X$ is contained in some $I_{K}$. Then for each $M<\omega$ the set

$$
A(K, M)=\bigcup_{-1 \leq n<K} \bigcup_{m<K} A_{n, m} \cup \bigcup_{-1 \leq n<K} \bigcup_{m>M} A_{n, m} \cup \bigcup_{n>M} \bigcup_{m<\omega} A_{n, m}
$$

equals $f^{-1}[U]$ for some open set $U \supseteq I_{K}$. So $(* *)$ follows from the next lemma.

Lemma 4.3. For every $K<\omega$ there are $N, M<\omega$ such that $e_{N}$ is equal to $u^{-1} v$ for no $u, v \in A(K, M)$. 
Proof. Fix $K<\omega$. Let $N=K+3$, so $N>2$. By Lemma 4.1, choose $M>N$ so large that

$$
e_{N} \neq u^{-1} \cdot v \text { for any } u, v \in \bigcup_{m<K} A_{-1, m} \cup \bigcup_{m>M} A_{-1, m} .
$$

We claim that $N$ and $M$ satisfy our requirements in the lemma. Suppose for contradiction that $e_{N}=u^{-1} v$ for some $u, v \in A(K, M)$. We will show this is impossible by checking all cases.

CASE 1: Both $u, v$ are special. Then $e_{N} \neq u^{-1} v$ by the choice of $M$.

CASE 2: Both $u, v$ are common. Then $e_{N}=u^{-1} \cdot v$ implies one of $u, v$ must either begin or end with $e_{N}^{ \pm 1}$. This is impossible by the choice of $N, M$.

CASE 3(a): $u$ is special, $v$ is derived. Then $u$ is an initial segment of $v$ or vice versa. In the first case $v$ is put into $A_{N, N}$ at Step 3(a) of the construction of $A_{n, m}$ 's, so $v \notin A(K, M)$, a contradiction. The second case is impossible by the choice of $w_{k}, k \geq 0$.

CASE 3(b): $v$ is special, $u$ is derived. Similar to Case 3(a).

Case 3(c): Both u, $v$ are derived. Similar to Case 3(a).

CASE 4. One of $u, v$ is common and the other is special or derived. A similar check.

Actually, we have obtained an example of an $\aleph_{0}$-saturated group $H$ covered by countably many 0-type-definable sets $X_{\alpha}, \alpha \in X$, such that $H \backslash X_{\omega}^{-1} \cdot X_{\omega}$ is countable and $H \neq X_{I}^{-1} \cdot X_{I}$ for every finite set $I \subseteq X$. If we replace the sets $X_{\alpha}$ by $X_{\alpha}^{-1}$, we get a covering such that $H \backslash X_{\omega} \cdot X_{\omega}^{-1}$ is countable and $H \neq X_{I} \cdot X_{I}^{-1}$ for every finite set $I \subseteq X$.

5. Coverings of types. In this section we consider a complete type $p(x) \in S(\emptyset)$ in a complete first-order theory $T$. We work in a monster model $\mathfrak{C}$ of $T$. We assume that $p(\mathfrak{C}) \times p(\mathfrak{C})$ is covered by countably many 0 -typedefinable sets $X_{n}, n<\omega$. At the level of types this means that the space $P=S_{x y}(\emptyset) \cap[p(x) \cup p(y)]$ is covered by countably many closed sets $P \cap$ $\left[X_{n}(x, y)\right], n<\omega$. In this case, as mentioned in the introduction, in [NP] we have proved that

$\left(\mathrm{C}^{\prime}\right) \quad$ any $a, b \in p(\mathfrak{C})$ may be connected by a path $a, c_{1}, c_{2}, b$ of length 3 so that the edges $\left\langle a, c_{1}\right\rangle,\left\langle c_{1}, c_{2}\right\rangle,\left\langle b, c_{2}\right\rangle$ lie in $X_{<n}$ for some $n<\omega$.

This statement and its proof are closely related to (C1). In Section 1 we gave a new proof of (C1) (see Corollary 1.7), using (weak) generic types in a group. Here we give a new proof of $\left(\mathrm{C1}^{\prime}\right)$, using a new notion of (weakly) c-free extensions, generalizing non-forking extensions in the stable case, and 
corresponding to (weak) generic types in a group. To explain this correspondence notice that to define (weak) generic sets and types in a group all we needed was just the transitive action of $G$ on itself by left translations. Here instead we have the action of the group $\operatorname{Aut}(\mathfrak{C})$ on $\mathfrak{C}$, which is transitive on $p(\mathfrak{C})$. This leads to the following definition, corresponding to Definition 1.1. For $U \subseteq \mathfrak{C}$, the images of $U$ under the automorphisms of $\mathfrak{C}$ [fixing $A \subseteq \mathfrak{C}$ ] are called the conjugates of $U$ [over $A$ ].

Definition 5.1. We say that a set $U \subseteq \mathfrak{C}$ is $c$-free over $p$ if $p(\mathfrak{C})$ is covered by finitely many conjugates of $U$. We say that a formula $\varphi(x)$ is $c$-free over $p$ if the set $\varphi(\mathfrak{C})$ is c-free over $p$. We say that a type $q(x)$ is $c$-free over $p$ if every formula $\varphi(x)$ with $q(x) \vdash \varphi(x)$ is c-free over $p$.

The $\mathrm{c}$ in "c-free" stands for "covering". In the stable case, a formula $\varphi(x)$ is c-free over $p$ iff $p(x) \cup\{\varphi(x)\}$ does not fork over $\emptyset$, and a formula $\varphi(x)$ does not fork over $\emptyset$ iff $\varphi(x)$ is c-free over some $q(x) \in S(\emptyset)$. This follows e.g. from the open mapping theorem (see [Pi] for the background on forking in stable theories). Hence in the stable case c-free $=$ non-forking, and complete c-free extensions of $p$ exist over any set $A \subseteq \mathfrak{C}$.

In the simple and o-minimal cases this is not so. One could say that non-forking loses there some of its covering properties from the stable case. In particular, complete c-free extensions may not exist (see e.g. the random graph and the o-minimal structure of the reals). As in Section 1, to overcome this drawback we weaken the definition.

DeFinition 5.2. We say that a set $U \subseteq \mathfrak{C}$ is weakly c-free over $p$ if $U \cup V$ is c-free over $p$ for some $V \subseteq \mathfrak{C}$ that is not c-free over $p$. We say that a formula $\varphi(x)$ is weakly $c$-free over $p$ if the set $\varphi(\mathfrak{C})$ is. A type $q(x)$ is called weakly $c$-free over $p$ if every formula $\varphi(x)$ with $q(x) \vdash \varphi(x)$ is weakly c-free over $p$.

We are interested in the definable weakly c-free sets (or even in relatively definable subsets of $p(\mathfrak{C}))$. The next lemma provides an alternative definition in this case.

Lemma 5.3. Assume $U \subseteq \mathfrak{C}$ is definable. Then the following conditions are equivalent.

(1) $U$ is weakly c-free over $p$.

(2) For some finitely many conjugates $U_{i}, i<n$, of $U$ the set $\bigcap_{i<n} U_{i}^{\mathrm{c}}$ is not c-free over $p$.

(3) For some definable set $V \subseteq \mathfrak{C}$ that is not c-free over $p$, the union $U \cup V$ is c-free over $p$.

Proof. See the proof of Lemma 1.3.

Notice that Lemma 5.3 could also be formulated for relatively definable subsets of $p(\mathfrak{C})$. The next lemma corresponds to Lemma 1.4. 


\section{LEMMA 5.4.}

(1) If $U_{1}, U_{2} \subseteq \mathfrak{C}$ are not weakly c-free over $p$, then $U_{1} \cup U_{2}$ is not weakly $c$-free over $p$.

(2) If $q(x)$ is a (partial) type over $A \subseteq \mathfrak{C}$ that is weakly $c$-free over $p$, then some $q^{\prime}(x) \in S(A)$ extending $q(x)$ is weakly c-free over $p$. Necessarily, $p(x) \subseteq q^{\prime}(x)$.

Let

$$
S_{\mathrm{wcf}, p}(A)=\{q \in S(A): q \text { is weakly c-free over } p\} .
$$

We see that $S_{\mathrm{wcf}, p}(A)$ is closed and non-empty. The next lemma corresponds to Lemma 1.5 .

LEMMA 5.5 .

(1) Assume $M$ is $\aleph_{0}$-saturated. If some type in $S_{\mathrm{wcf}, p}(M)$ is c-free over $p$, then they all are.

(2) If there is just one type in $S_{\mathrm{wcf}, p}(A)$, then it is c-free over $p$.

Proof. (1) Suppose some type $q(x) \in S_{\mathrm{wcf}, p}(M)$ is not c-free over $p$. This means that some $M$-definable set $U \subseteq \mathfrak{C}$ that is c-free over $p$ can be divided into two $M$-definable sets $A, B$ that are not c-free over $p$. Say, $U=\varphi(\mathfrak{C}, m)$, $A=\psi(\mathfrak{C}, m)$ and $B=\chi(\mathfrak{C}, m)$ for some formulas $\varphi, \psi, \chi$ and some $m \subseteq M$. Then there are some finitely many conjugates $m_{i} \subseteq M, i<n$, such that $p(x) \vdash \bigvee_{i<n} \varphi\left(x, m_{i}\right)$. We will show that any type $q^{\prime} \in S_{\mathrm{wcf}, p}(M)$ is not c-free over $p$.

Since $p(x) \subseteq q^{\prime}(x)$, we see that $q^{\prime}(x) \vdash \varphi\left(x, m_{i}\right)$ for some $i<n$. Then either $q^{\prime}(x) \vdash \psi\left(x, m_{i}\right)$ or $q^{\prime}(x) \vdash \chi\left(x, m_{i}\right)$. Neither $\psi\left(x, m_{i}\right)$ nor $\chi\left(x, m_{i}\right)$ is c-free over $p$, hence $q^{\prime}$ is not either.

(2) A similar proof.

As an example, notice that if $T$ is o-minimal, then for any set of parameters $A$, any non-algebraic type $p(x) \in S_{1}(\emptyset)$ has just two extensions in $S_{\mathrm{wcf}, p}(A)$, and none of them is c-free over $p$.

We can define (weakly) c-free formulas over, and extensions of, a type $p^{\prime} \in S(A)$ for any $A \subseteq \mathfrak{C}$. Then we can say that a formula $\varphi(x)$ is (weakly) c-free over $A$ if $\varphi(x)$ is (weakly) c-free over some $p^{\prime}(x) \in S(A)$. However, the notion of weak c-freeness does not, in general, have the nice properties of non-forking from the stable or simple case.

Recall that $P$ denotes the space $S_{x y}(\emptyset) \cap[p(x) \cup p(y)]$. For any $S \subseteq P$ and $a \models p$ let

$$
S_{a}=\left\{q(a, y) \in S_{y}(a): q(a, y) \in S\right\} .
$$

In particular, $P_{a}=S_{y}(a) \cap[p(y)]$. Notice that $S_{a}$ is relatively open in $P_{a}$ iff $S$ is relatively open in $P$. Let

$$
P_{\mathrm{wcf}}=\{q(x, y) \in P \text { : for } a \models p, q(a, y) \text { is weakly c-free over } p\} \text {. }
$$


Then $P_{\text {wcf }}$ is closed, since its complement in $P$ is relatively open in $P$. Notice that in general $q(x, y) \in P_{\text {wcf }}$ does not imply $q(y, x) \in P_{\text {wcf }}$, since weak c-freeness is not always symmetric. The next proposition corresponds to Proposition 1.6.

Proposition 5.6. Assume $S \subseteq P_{\text {wcf }}$ is non-empty and relatively open. Then there are some finitely many $c_{i} \models p, i<k$, such that for every $b \models p$ there is some $d \models p$ such that $\operatorname{tp}(b, d) \in S$ and $\operatorname{tp}\left(c_{i}, d\right) \in S$ for some $i<k$.

Proof. In this proof a "definable set" will mean a "relatively definable subset of $p(\mathfrak{C})$ or of $p(\mathfrak{C}) \times p(\mathfrak{C})$ ". We identify definable sets with the formulas that (relatively) define them.

Choose a non-empty 0-definable set $U \subseteq p(\mathfrak{C}) \times p(\mathfrak{C})$ with

$$
S \supseteq P_{\mathrm{wcf}} \cap[U(x, y)] \text {. }
$$

We can assume that $S=P_{\text {wcf }} \cap[U(x, y)]$. Hence for any $c \models p, U(c, y)$ is weakly c-free over $p$. Choose a (relatively) definable set $V=V(e, \mathfrak{C}) \subseteq p(\mathfrak{C})$ that is not c-free over $p$, but $U \cup V$ is c-free over $p$. Choose a finite set $\left(c_{i}, e_{i}\right)$, $i<k$, of conjugates of $(c, e)$ such that

$$
p(y) \vdash \bigvee_{i<k}\left(U\left(c_{i}, y\right) \vee V\left(e_{i}, y\right)\right) .
$$

As in the proof of Proposition 1.6 we show that

$$
\text { the set }(U(c, \mathfrak{C}) \cup V(e, \mathfrak{C})) \backslash S(c, \mathfrak{C}) \text { is not c-free over } p \text {. }
$$

To finish the proof, we show that for every $b \models p$ there is some $d \in S(b, \mathfrak{C})$ with $d \in \bigcup_{i<k} S\left(c_{i}, \mathfrak{C}\right)$.

If not, then there is some $b \models p$ such that $d \notin \bigcup_{i<k} S\left(c_{i}, \mathfrak{C}\right)$ for every $d \in S(b, \mathfrak{C})$. Still $d \in \bigcup_{i<k}\left(U\left(c_{i}, \mathfrak{C}\right) \cup V\left(e_{i}, \mathfrak{C}\right)\right)$, and hence

$$
S(b, \mathfrak{C}) \subseteq \bigcup_{i<k}\left(\left(U\left(c_{i}, \mathfrak{C}\right) \cup V\left(e_{i}, \mathfrak{C}\right)\right) \backslash S\left(c_{i}, \mathfrak{C}\right)\right)
$$

Choose $e_{k}$ so that for $c_{k}=b,\left(c_{k}, e_{k}\right)$ and $(c, e)$ are conjugate. Then we see that

$$
U\left(c_{k}, \mathfrak{C}\right) \cup V\left(e_{k}, \mathfrak{C}\right) \subseteq \bigcup_{i \leq k}\left(\left(U\left(c_{i}, \mathfrak{C}\right) \cup V\left(e_{i}, \mathfrak{C}\right)\right) \backslash S\left(c_{i}, \mathfrak{C}\right)\right),
$$

hence the set on the right hand side is c-free over $p$. Thus so also is the set $(U(c, \mathfrak{C}) \cup V(e, \mathfrak{C})) \backslash S(c, \mathfrak{C})$, contradicting $(*)$.

Corollary 5.7. Assume $S \subseteq P_{\mathrm{wcf}}$ is non-empty and relatively open. Then there are some finitely many types $r_{i}(x, y) \in P, i<k$, such that for every $a, b \models p$ there are $c, d \models p$ with $\operatorname{tp}(a, c) \in\left\{r_{i}: i<k\right\}$ and 
$\operatorname{tp}(c, d), \operatorname{tp}(b, d) \in S$. Moreover, for every $a \models p$ we can choose $c_{i}=r_{i}(a, y)$, $i<k$, so that for every $b \models p$ there are $c, d$ as above so that additionally $c \in\left\{c_{i}: i<k\right\}$.

Proof. Let $c_{0}, \ldots, c_{k-1}$ be as in the proof of Proposition 5.6. Let $r_{i}=$ $\operatorname{tp}\left(c_{0}, c_{i}\right)$. Now consider an arbitrary $a \models p$. Applying an automorphism of $\mathfrak{C}$ we can assume $c_{0}=a$. Then by Proposition 5.6 we are done.

The next corollary was proved in $[\mathrm{NP}]$. Here we give a different proof.

Corollary 5.8. Assume $P$ is covered by countably many closed sets $X_{n}, n<\omega$. Then there are some finitely many types $r_{i} \in P, i<k$, and a natural number $n$ such that for every $a, b \models p$ there are $c, d \models p$ with $\operatorname{tp}(a, c) \in\left\{r_{i}, i<k\right\}$ and $\operatorname{tp}(c, d), \operatorname{tp}(b, d) \in X_{n}$.

Proof. By the Baire category theorem some of the sets $X_{n} \cap P_{\text {wcf }}$ have non-empty relative interior in $P_{\text {wcf }}$, so Corollary 5.7 applies.

In the case of a simple theory, weakly c-free extensions are related to non-forking extensions.

LEMMA 5.9. Assume $T$ is simple and $\varphi(x, a)$ is weakly $c$-free over $p \in$ $S(\emptyset)$. Then $\varphi(x, a)$ does not fork over $\emptyset$.

Proof. Choose a formula $\psi(x, b)$ such that $\psi$ is not c-free over $p$ and $\varphi(x, a) \vee \psi(x, b)$ is c-free over $p$. So for some $f_{i} \in \operatorname{Aut}(\mathfrak{C}), i<k$, we have $p(x) \vdash \varphi^{\prime}\left(x, a^{\prime}\right) \vee \psi^{\prime}\left(x, b^{\prime}\right)$, where

$$
\varphi^{\prime}\left(x, a^{\prime}\right)=\bigvee_{i<k} \varphi\left(x, f_{i}(a)\right) \quad \text { and } \quad \psi^{\prime}\left(x, b^{\prime}\right)=\bigvee_{i<k} \psi\left(x, f_{i}(b)\right) .
$$

Then $\psi^{\prime}\left(x, b^{\prime}\right)$ is still not c-free over $p$. Suppose $\varphi(x, a)$ forks over $\emptyset$. Then $\varphi^{\prime}\left(x, a^{\prime}\right)$ also forks over $\emptyset$, hence it divides over $\emptyset$. Thus for some $g_{j} \in \operatorname{Aut}(\mathfrak{C})$, $j<l$, the set $\left\{\varphi^{\prime}\left(x, g_{j}\left(a^{\prime}\right)\right): j<l\right\}$ is inconsistent. We see that

$$
p(x) \vdash \bigvee_{j<l} \psi^{\prime}\left(x, g_{j}\left(b^{\prime}\right)\right),
$$

hence $\psi^{\prime}\left(x, b^{\prime}\right)$ is c-free over $p$, a contradiction.

Again, we do not know a simple theory where weak c-freeness $\neq$ nonforking.

6. Open problems. We end this paper with a set of problems and questions.

Problem 1. Find a group definable in a simple theory where weak generic $\neq \mathrm{f}$-generic, or prove that in some cases still weak generic $=\mathrm{f}$-generic. 
Problem 2. The first application of generic types was the fact that a stable group $G$ with regular generic types is abelian-by-finite [Po]. Is there a counterpart of this result for weak generic types?

Problem 3. Find a simple theory where weak c-freeness $\neq$ non-forking, or prove that in some cases still weak c-freeness $=$ non-forking.

Problem 4. Determine the number $k$ of steps needed to generate the group $G$ (defined in the introduction) in the situation where $G$ is definable in an o-minimal or simple theory. As we know, there are two possibilities: either $k=2$ or $k=2.5$. Is it true that in the o-minimal case $k=2$ holds? The crucial point here may be to realize how closely we may describe (by a type-definable set) a free subgroup of $G$.

Problem 5. Assume $G$ is an $\aleph_{0}$-saturated group, covered by countably many sets $X_{n}, n<\omega$. Corollary 1.7 says that in this case $G$ is generated by finitely many of them, in finitely many steps. Is this still true if $X_{n}$ are Borel? Can we still find a bound on the number of steps needed to generate $G$ (maybe depending on the Borel class of the sets $X_{n}$ )? This seems related to the question of how the notion of first Baire category is related to the group operation of $G$. In general, our results on $\aleph_{0}$-saturated groups show some similarity to those for compact topological groups.

Assume $G$ is a compact topological group, covered by countably many Borel sets $X_{n}, n<\omega$. Then they have the Baire property (and are also measurable with respect to the Haar measure). Hence there is some $n<\omega$ and some open non-empty set $U \subseteq G$ such that $U \backslash X_{n}$ is meager. It is easy to see that then $G$ is generated by some finitely many of $X_{n}$ 's in finitely many steps.

Problem 6. Strengthen Theorem 2.4 to make it hereditary. That is, define a property $C$ of subsets of an $\aleph_{0}$-saturated group $G$ such that if a type-definable set $X \subseteq G$ satisfies $C$, then $G=X \cdot X^{-1}$, and if $X$ is split into countably many type-definable sets $X_{n}, n<\omega$, then for some $n$ also $X_{<n}$ satisfies $C$.

\section{References}

[Be] G. Bergman, Generating infinite symmetric groups, preprint, 2004.

[Ne1] L. Newelski, M-gap conjecture and m-normal theories, Israel J. Math. 106 (1998), $285-311$.

[Ne2] - The diameter of a Lascar strong type, Fund. Math. 176 (2003), 157-170.

[NP] L. Newelski and M. Petrykowski, Coverings of groups and types, J. London Math. Soc. (2) 71 (2005), 1-21.

[Pi] A. Pillay, Geometric Stability Theory, Clarendon Press, Oxford, 1996.

[Po] B. Poizat, Groupes stables, avec types génériques réguliers, J. Symbolic Logic 48 (1983), 339-355. 
[Wa] F. Wagner, Simple Theories, Kluwer, Dordrecht, 2000.

[W] S. Wagon, The Banach-Tarski Paradox, Cambridge Univ. Press, Cambridge, 1985.

Institute of Mathematics

University of Wrocław

Pl. Grunwaldzki 2/4

50-384 Wrocław, Poland

E-mail: newelski@math.uni.wroc.pl

petryk@math.uni.wroc.pl

Received 3 December 2004;

in revised form 25 April 2006 